\title{
PEGylation affects the self-assembling behavior of amphiphilic octapeptides
}

\author{
Diego Romano Perinelli ${ }^{1}$, Mario Campana ${ }^{2}$, Ishwar Singh ${ }^{3}$, Driton Vllasaliu ${ }^{4}$, James Doutch ${ }^{2}$, \\ Giovanni Filippo Palmieri ${ }^{1}$, Luca Casettari*5
}

\author{
${ }^{1}$ School of Pharmacy, University of Camerino, via Gentile III da Varano, 62032 Camerino, MC, Italy. \\ ${ }^{2}$ ISIS Neutron Facility, Science and Technology Facilities Council, Rutherford Appleton Laboratory, Didcot OX11 \\ OQX, United Kingdom \\ ${ }^{3}$ School of Pharmacy, University of Lincoln, Green Lane, Lincoln, LN6 7DL, UK \\ ${ }^{4}$ King's College London, Institute of Pharmaceutical Science, London SE1 9NH, UK \\ ${ }^{5}$ Department of Biomolecular Sciences, University of Urbino Carlo Bo, Piazza del Rinascimento, 6, 61029 Urbino \\ (PU), Italy.
}

\section{ABSTRACT}

Surfactant-like peptides are a class of amphiphilic macromolecules, which are able to self-assemble in water forming different supramolecular structures. Among them, octapeptides composed of six hydrophobic and two hydrophilic residues have attracted interest since they have a length similar to those of natural phospholipids. Supramolecular structures of different amphiphilic octapeptides have been widely reported, but no study has been performed aimed at investigating the effect of PEGylation on their self-assembling behaviour. The aim of the present work was to synthesize and characterise the self-assembling behaviour of PEGylated alanine- or valine based amphiphilic

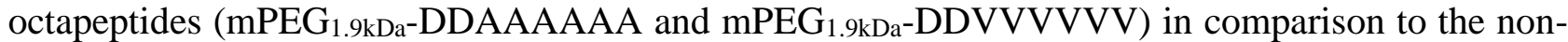
PEGylated ones (DDAAAAAA and DDVVVVVV).

The self-aggregation process in ultrapure water was investigated by fluorescence spectroscopy, small angle neutron scattering (SANS), dynamic light scattering (DLS), while the secondary structure was assessed by circular dichroism.

PEGylation markedly affects the self-assembling behaviour of these amphiphilic octapeptides in terms of both critical aggregation concentration (CAC) and shape of the formed supramolecular aggregates. Indeed, PEGylation increases $\mathrm{CAC}$ and prevents the self-aggregation into fibrillary supramolecular aggregates (as observed for non-PEGylated peptides), by promoting the formation of micelle-like structures (as demonstrated for valine-based octapeptide). 

37

38 39 40 41 42 43 44 45 46 47 48 49 50 51 52 53 54 55 56 57 58 59 60 61 62 63 64 65 66 67 


\section{INTRODUCTION}

In recent years, molecular self-assembly of peptides has attracted great interest due to wide potential applications in nanotechnology and nanomedicine for the design of nanostructured smart materials ${ }^{1}$. The amphiphilic self-assembled peptides are termed as "surfactant-like peptides". They are composed of a sequence of consecutive hydrophobic amino acids as the tail and one or two hydrophilic amino acids as the head ${ }^{2}$. Generally, the hydrophobic sequences contain glycine $(G)$, alanine (A), valine (V), leucine (L) or isoleucine (I) residues, while the hydrophilic heads are made up of negatively (i.e. aspartic (D) or glutamic acid (E)) or positively (i.e. lysine (K), histidine (H) or arginine (R)) charged amino acids. The hydrophobic tail of a surfactant-like peptide is composed of six to nine hydrophobic residues, so that it is similar to the length of natural phospholipids (around 2.5-3 nm).

Valine and alanine residues are preferred to glycine, leucine and isoleucine residues since they produce more homogeneous and stable structures, probably due to the formation of stronger $\beta$-sheet elements ${ }^{3}$.

The self-assembling properties of these materials can be tuned by changing the peptide sequences, both in the hydrophobic and hydrophilic region, giving a range of supramolecular architectures, including nanotubes, nanovesicles or nanofibers. Upon dissolution in water (4-5 $\mathrm{mM})$, negatively charged peptides, such as Ac-A6D, Ac-V6D, Ac-G8DD or KV6, are able to form open-ended nanotubes with 30-50 $\mathrm{nm}$ of diameter and different lengths up to $1 \mu \mathrm{m}^{4}$.

The amphiphilic sequences consisting of alanine, valine and glutamic acid can be coupled with other materials to produce nanostructures with a wide range of applications. In one such example, these sequences were coupled with a palmitoyl chain to form fibrillar nanostructures as promising biomimetic hydrogel scaffolds 5 . In fact, Pashuck et al. observed that the arrangement of the hydrophobic residues not only affects the supramolecular structures but also the stiffness of the obtained hydrogel as a function of the total number of the hydrophobic residues and the strength of the $\beta$-sheet regions. The functionalization with lipids was also found to increase the in vivo stability of peptides.

Apart from the modification with lipids, no other chemical functionalization of these alanine, valine or glutamic based amphiphilic peptides is reported in the literature.

PEGylation is a common and well known strategy to improve water solubility and stability of peptides, and PEGylated peptides offer other advantages following in vivo administration, including increased plasma half-life and reduced immunogenity ${ }^{6}$.

Different studies were recently performed on a tetra-phenylalanine peptide linked to PEGs with different molecular weights ( $5 \mathrm{kDa}, 1.8 \mathrm{kDa}, 1.2 \mathrm{kDa}$ and $350 \mathrm{Da})$ to investigate the self-assembling behaviour in aqueous solution ${ }^{7-10}$. As an example, amphiphilic 3-helix PEGylated lipopeptides were 
synthesized and were found to self-assemble into monodisperse micellar nanoparticles of $15 \mathrm{~nm}$ diameter. After loading with doxorubicin, used as model anticancer drug, they showed minimal leakage after $12 \mathrm{~h}$ of incubation with serum proteins at $37^{\circ} \mathrm{C}$. Moreover, the in vivo half-life was 29.5 hours and minimal accumulation in the liver and spleen was observed using positron emission tomography ${ }^{11}$.

Despite the well-established beneficial attributes and improved performances imparted by PEGylation on different peptides, no study has been focused on the effect of PEGylation on the selfassembling behaviour of surfactant-like octapeptides. Thus, the aim of this work was to synthesize and investigate for the first time the self-assembling behaviour in water of PEGylated alanine- and valine-based surfactant-like octapeptides $\quad\left(\mathrm{mPEG}_{1.9 \mathrm{kDa}}-\mathrm{DDAAAAAA}\right.$ and $\mathrm{mPEG}_{1.9 \mathrm{kDa}^{-}}$ DDVVVVVV) and to compare them with the non-PEGylated ones (DDAAAAAA and DDVVVVVV).

\section{EXPERIMENTAL SECTION}

Synthesis of amphiphilic octapeptides

All peptides were synthesized through the solid phase automated microwaved-assisted approach starting from protected amino acid Fmoc-ALA-OH (Fluorochem, UK), Fmoc-VAL-OH (Fluorochem, UK) Fmoc-ASP(otbu)-OH (Fluorochem, UK). Rink amide ChemMatrix (Biotage, NC, USA) was used as resin. For the amino acid sequence, the coupling agents were Ethyl 2-cyano-2 (hydroxyimino) acetate (Oxyma; Fluorochem, UK) and N, N'-Diisopropylcarbodiimide (DIC; Fluorochem, UK). Coupling were performed at $75{ }^{\circ} \mathrm{C}$ for 5 minutes from the first to the fourth amino acid, for 7 minutes from the fifth and sixth amino acid and for 10 minutes for the last two amino acids. Subsequently, carboxylic-ended poly(ethylene glycol) monomethyl ether (1.9 kDa) was coupled to the amino end of the amino acid sequence attached to the resin. Carboxylic-ended poly(ethylene glycol) monomethyl ether was synthesized from mPEG (1.9 kDa; Polysciences Europe, Germany). For the synthesis, mPEG was functionalised on the free hydroxyl group with glutaric anhydride (Sigma Aldrich, Italy), using 4-Dimethylaminopyridine (DMAP, Sigma Aldrich, Italy) as organocatalyst, in dichloromethane, to obtain a carboxylic end. After the reaction, the polymer was purified by dialysis using $1 \mathrm{kDa}$ MWCO membrane (Spectra/Por ${ }^{\circledR}$, USA).

Carboxylic-ended poly(ethylene glycol) monomethyl ether was characterised by ${ }^{1} \mathrm{H}-\mathrm{NMR}$ and gel permeation chromatograpy (GPC) analysis $\left(\mathrm{M}_{\mathrm{n}} 1842 ; \mathrm{M}_{\mathrm{w}} 2005 ;\right.$ PDI 1,088). The Carboxylic-ended poly(ethylene glycol) monomethyl ether coupling was performed using 1- 
136 [Bis(dimethylamino)methylene]-1H-1,2,3-triazolo[4,5-b]pyridinium 3-oxid hexafluorophosphate 137 (HATU, Sigma-Aldrich, UK) and N,N-Diisopropylethylamine (DIPEA; Sigma-Aldrich, UK) at 75 $138{ }^{\circ} \mathrm{C}$ for 10 minutes. After cleavage from the resin with trifluoroacetic acid (Fluorochem, UK), peptides were precipitated in cold diethyl ether. The raw materials were purified through RP-HPLC (Thermo Scientific Dionex Ultimate 3000 RP-HPLC equipped with a Phenomenex Gemini NX C18 110 A, $150 \times 4.6 \mathrm{~mm}$ ) column, using the following buffer systems: A: $50 \mathrm{mM}$ ammonium carbonate, B: acetonitrile and a flow rate of $1 \mathrm{ml} / \mathrm{min}$. The following gradient was applied: $95 \%$ A for $2 \mathrm{~min}$; 5-

Mass analysis

For electron spray mass ionization (ESI) analysis, approximately $1 \mathrm{mg}$ of each peptide was dissolved in ultrapure water and analysed by direct injection in an ESI mass apparatus (HP 1100 LC/MSD, Agilent) equipped with a single quadrupole detector. The sample was analysed in the positive mode at a fragmentor voltage of $30 \mathrm{~V}$.

MALDI-TOF spectra were acquired on a 400 Plus MALDI TOF/TOF Analyzer (AB Sciex, MA, USA). Peptides were dissolved in ultrapure water with $0.05 \%$ TFA and mixed, in the same volumetric amount, with the saturated matrix solution (2, 5-Dihydroxybenzoic acid saturated solution in 50:50, $0.05 \%$ TFA water/acetonitrile). $1 \mu \mathrm{L}$ of this solution was applied onto the MALDI sample plate and allowed to co-crystalize at room temperature and then analysed at a 6000 laser intensity.

Far UV CD spectra of peptides were collected using a $\pi^{*}-180$ step-flow spectrometer (Applied Photophysics, Leatherhead, UK). Peptides were analysed dissolved in ultrapure water at a concentration of $1 \mathrm{mg} / \mathrm{mL}$ for non-PEGylated and $3 \mathrm{mg} / \mathrm{mL}$ for PEGylated peptides. Spectra were recorded in the range of 180-280 $\mathrm{nm}$ with a step of $0.1 \mathrm{~nm}$ and a bandwidth of $1 \mathrm{~nm}$. The acquisition time was $2 \mathrm{~s}$ for each point. A $10 \mathrm{~mm}$ path length quartz cuvette was filled with the peptide solution and measured at room temperature.

Steady-state fluorescence spectra of pyrene in the presence of different concentration of nonPEGylated or PEGylated peptides were recorded at $37^{\circ} \mathrm{C}$ using a spectrofluorimeter (LS-55, PerkinElmer) equipped with a thermostated cell (HAAKE C25P thermostat). The fluorescence emission 
spectra $(350-600 \mathrm{~nm})$ of pyrene were measured using an excitation wavelength $\lambda_{\mathrm{exc}}=338 \mathrm{~nm}$ and $2.5 \mathrm{~nm}$ slits. The intensity ratio of the first (I) and third (III) vibronic band of the emission spectrum of pyrene, at $372 \mathrm{~nm}$ and $384 \mathrm{~nm}$, was plotted against the peptide concentration. The critical aggregation concentration (CAC) of peptides was determined by fitting the experimental data with the following equation (GraphPad Prism 6):

$$
Y=\frac{\text { Bottom }+(\text { Top }- \text { Bottom })}{1+10^{\wedge}[(\log C M C-x) * \text { Hill slope }}
$$

where Top and Bottom are the plateau of the curve in the unit of Y axis, hill slope is the steepness of the curve.

\section{Small angle neutron scattering (SANS)}

SANS data were collected on the LOQ small-angle diffractometer at the ISIS Pulsed Neutron Source (STFC Rutherford Appleton Laboratory, Didcot, U.K.), using a two-dimensional, position-sensitive, ${ }^{3} \mathrm{He}$ detector neutron detector to provide a simultaneous Q range of $0.08-2 \mathrm{~nm}^{-1} 12,13$. Each sample and solvent $\left(\mathrm{D}_{2} \mathrm{O}\right.$ for background) was placed in $2 \mathrm{~mm}$ path length quartz banjo cuvettes and measured for a total of 2 hours in order to gather data of high statistical precision. Each raw scattering data set was then corrected for the detector efficiencies, sample transmission and background scattering and converted to scattering cross-section data ( $\partial \Sigma / \partial \Omega$ vs Q) using the instrument-specific software (MantidPlot) ${ }^{14}$. Non-PEGylated peptides were analysed at $5 \mathrm{mg} / \mathrm{mL}$, while PEGylated peptides at 30 $\mathrm{mg} / \mathrm{mL}$ in ultrapure water at $37^{\circ} \mathrm{C}$. The temperature was controlled by using a thermostat (Julabo bath) with a precision of $0.1{ }^{\circ} \mathrm{C}$. SANS data were analysed using SASview 4.1 software.

\section{Dynamic light scattering (DLS)}

The hydrodynamic diameter (nm) and size distribution (width; $\mathrm{nm}$ ) of non-PEGylated and PEGylated peptides were determined by DLS from the size by intensity (\%) traces. DLS measurements were carried out using a Zetasizer Nano S (Malvern Instruments, UK) that employs a $173^{\circ}$ backscatter detector. For the analysis, samples were loaded inside disposable cuvette and equilibrated $300 \mathrm{~s}$ at the operating temperature. The operating settings were position $(\mathrm{mm}): 4.65$; attenuator: 11 and temperature: $37^{\circ} \mathrm{C}$. 


\section{RESULTS AND DISCUSSION}

All synthesized peptides were characterized by mass analysis. Mass analysis is a standard technique employed for the identification and determination of the eventual presence of impurities in the sample. ESI mass spectra (positive mode) indicate the purity of non-PEGylated peptides. In mass spectra, only one molecular ion $(\mathrm{m} / \mathrm{z})$ and the relative adduct ions can be recognized. For DDAAAAAA the molecular ion is at $674.5 \mathrm{~m} / \mathrm{z}$, while for DDVVVVVV the molecular ion is at 842.8 $\mathrm{m} / \mathrm{z}$ (Figure 1). These mass values are comparable to those theoretically calculated for these peptide sequences.

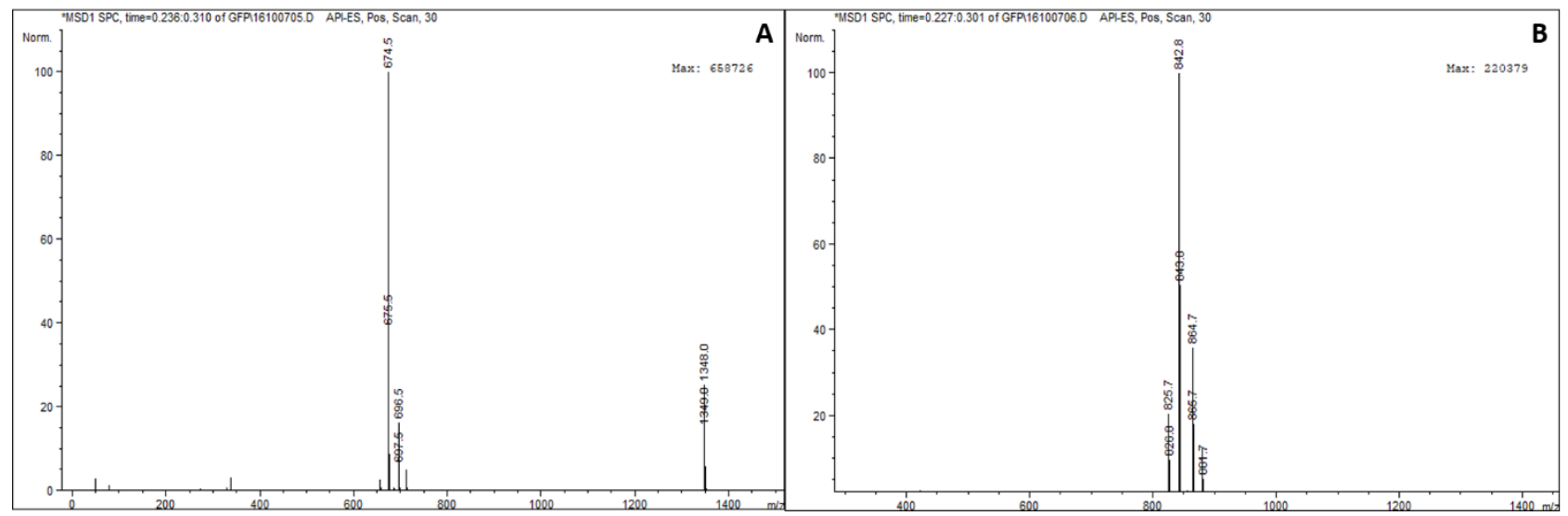

Figure 1 ESI mass spectra of non-PEGylated amphiphilic octapeptides: DDAAAAAA (A) and DDVVVVVV (B).

Due to the higher molecular weight (above $1000 \mathrm{Da}$ ), no reliable results were obtained from ESI mass analysis of PEGylated peptides. Specifically, a large number of fragment ions below 1000 Da were observed, which were not unambiguous assigned to the sample. Therefore, PEGylated peptides were subsequently analysed by MALDI-TOF. A single molecular weight distribution can be observed in the analysed mass range 1-5 $\mathrm{kDa}$ for both PEGylated peptides, referring to the PEGylated adducts, as demonstrated from the calculated average molecular weights (2778 Da for $\mathrm{mPEG}_{1.9 \mathrm{kDa}-\mathrm{DDAAAAAA}}$ and 2984 Da for mPEG 1.9 kDa-DDVVVVVV) (Figure 2). 

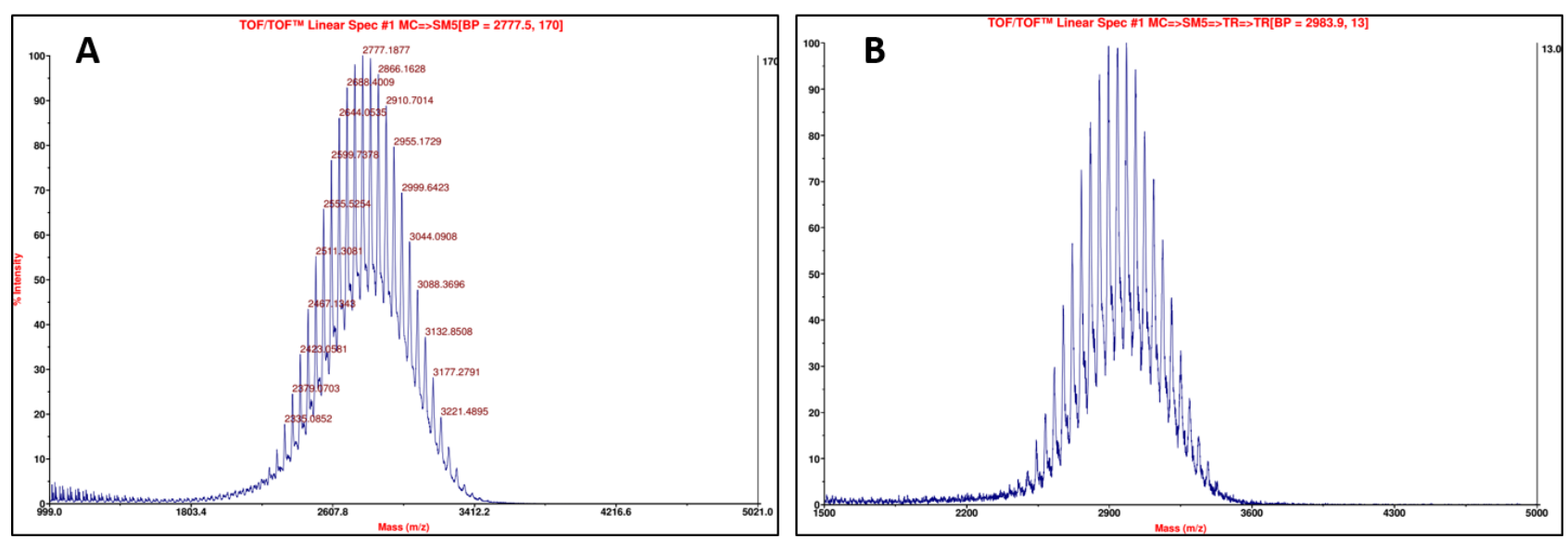

Figure 2 MALDI-TOF mass spectra of PEGylated amphiphilic octapeptides: $\mathrm{mPEG}_{1.9 \mathrm{kDa}^{-}}$

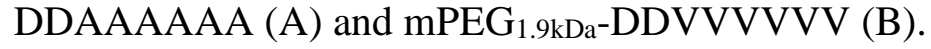

The secondary structure in aqueous dispersion of peptides was investigated by circular dichroism. As for other valine and alanine based-peptides ${ }^{15-17}$, this analysis confirmed that the amphiphilic peptides assume predominantly a beta-sheet conformation in water, as indicated by a minimum in ellipticity at around $220 \mathrm{~nm}$. An appreciable fraction of random coil was only observed for the non-PEGylated valine peptide (DDVVVVVV) due to the presence of a minimum at around 190-200nm. Random coil fraction was negligible for the other peptides. Notably, PEGylation does not affect the secondary structure of peptides since PEGylated amphiphilic octapeptides (DDAAAAAA and DDVVVVVV) maintains the beta-sheet conformation as the non-PEGylated ones (Figure 3).
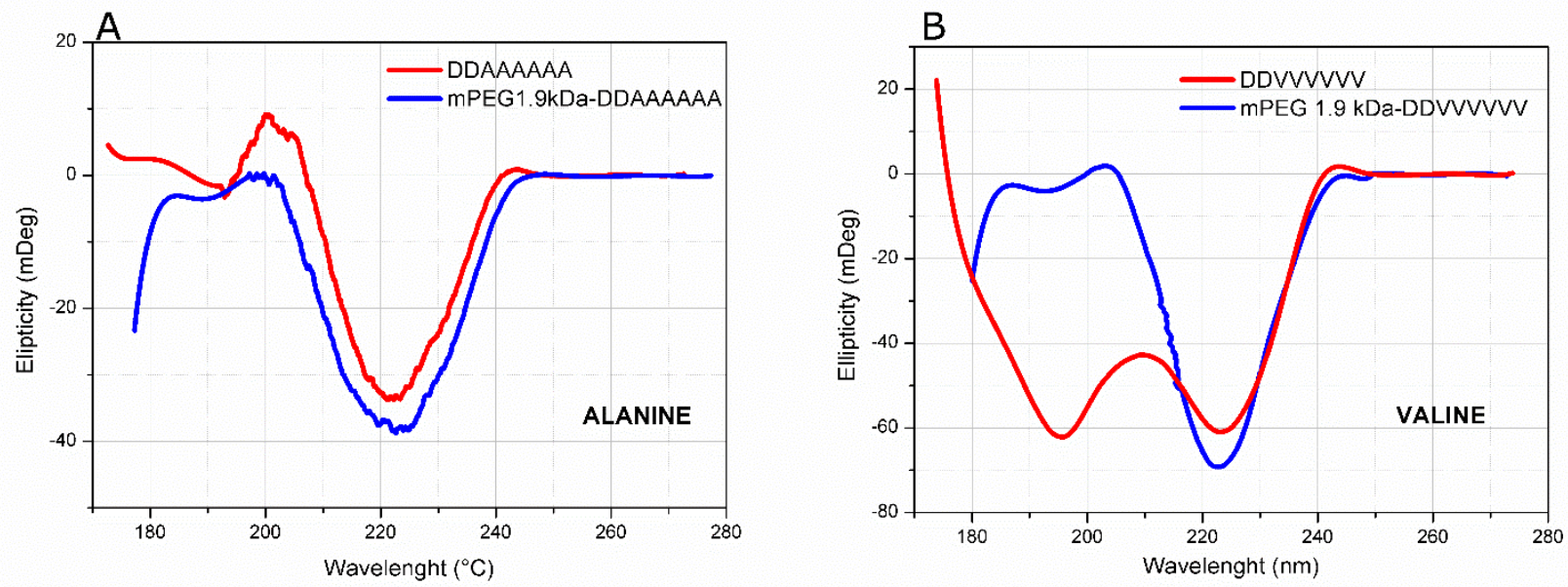

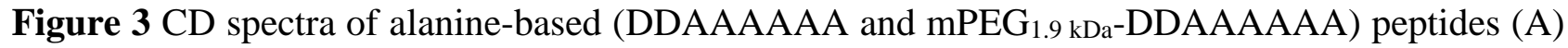
and valine-based (DDVVVVVV and $\mathrm{mPEG}_{1.9 \mathrm{kDa}-\mathrm{DDVVVVVV}}$ ) peptides (B). 
The critical aggregation concentration (CAC) values of the synthesized peptides were calculated by steady state fluorescence from the I/III peak ratio of pyrene, used as a probe. This ratio is particularly sensitive to the polarity of the microenvironment around the probe, which decreases at a peptide concentration at which the aggregation process begins. The calculated CAC values were $0.055 \pm$ $0.003 \mathrm{mg} / \mathrm{mL}\left(0.065 \mathrm{mM} ; \mathrm{R}^{2}=0.993\right)$ for DDVVVVVV; $0.906 \pm 0.047 \mathrm{mg} / \mathrm{mL}\left(1.3 \mathrm{mM} ; \mathrm{R}^{2}=0.982\right)$ for DDAAAAAA; $1.638 \pm 0.402 \mathrm{mg} / \mathrm{mL}\left(0.6 \mathrm{mM} ; \mathrm{R}^{2}=0.994\right)$ for $\mathrm{mPEG}_{1.9 \mathrm{kDa}-\mathrm{DDVVVVVV}}$. No changes in the I/III fluorescence ratio were observed for $\mathrm{mPEG}_{1.9 \mathrm{kDa} a}$-DDAAAAAA at the tested concentrations, suggesting no aggregation process. The results clearly indicated the effect of peptide sequence and PEG-coupling on CAC. Particularly, the lower CAC values for DDVVVVVV peptide in comparison to DDAAAAAA peptides are related to the higher hydrophobicity of valine residues. On the other hand, PEGylation has an opposite effect by inducing an increase in the self-aggregation concentration of the analysed peptides. The lower fluorescence intensity I/III ratio reached for concentrations of non-PEGylated peptides above CAC, in comparison to $\mathrm{mPEG}_{1.9 \mathrm{kDa}-\mathrm{DDVVVVVV}}$, indicates a more hydrophobic environment of the core compared to that of aggregates formed by the analysed PEGylated peptide (Figure 4).

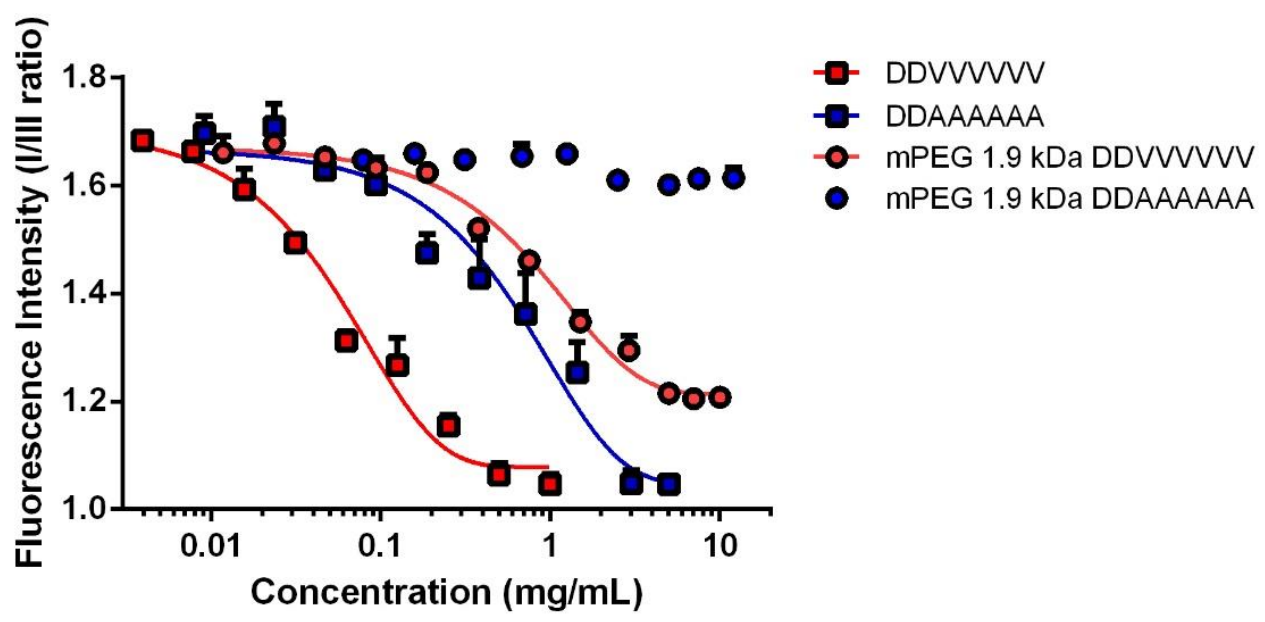

Figure 4 Variation of fluorescence intensity for the ratio between vibrionic peak I ( $\lambda 372 \mathrm{~nm})$ and peak III $(\lambda 384 \mathrm{~nm})$ of pyrene as a function of concentration for non-PEGylated (DDAAAAAA and DDVVVVVV) and PEGylated (mPEG ${ }_{1.9 \mathrm{kDa}-\mathrm{DDAAAAAA}}$ and $\left.\mathrm{mPEG}_{1.9 \mathrm{kDa}}-\mathrm{DDVVVVVV}\right)$ octapeptides.

The fitting of SANS profiles related to the PEGylated peptides, using the ellipsoid model, revealed the formation in aqueous dispersion of micellar-type aggregates with an elongated shape. The calculated radii were $5.1 \mathrm{~nm}$ (polar) and $11.7 \mathrm{~nm}$ (equatorial) for $\mathrm{mPEG}_{1.9 \mathrm{kDa}-\mathrm{DDVVVVVV}}$ and 1.8 
$\mathrm{nm}$ (polar) and $1.7 \mathrm{~nm}$ (equatorial) for $\mathrm{mPEG}_{1.9 \mathrm{kDa}}$-DDAAAAAA. This result highlighted a selfassembling process only for the PEGylated valine-based peptide and not for the alanine-based peptide at the tested concentration, confirming the results obtained from fluorescence spectroscopy using pyrene as a probe. On the contrary, non-PEGylated peptides showed a different scattering profiles which can be attributed to fibrillary structures of larger dimensions. DDVVVVVV profiles can be fitted with a lamellar model with a thickness of $2.8 \mathrm{~nm}$, while for DDAAAAAA the lamellar thickness was around $12.0 \mathrm{~nm}$. The higher computed polydispersity for DDAAAAAA with respect to DDVVVVVV peptides indicates the formation of less homogeneous structures for alanine basedpeptides (Figure 5A). The $\mathrm{mPEG}_{1.9 \mathrm{kDa}-\mathrm{DDVVVVV}}$ peptide was also analysed at different concentrations above CAC (30 mg/mL, $10 \mathrm{mg} / \mathrm{mL}$ and $3 \mathrm{mg} / \mathrm{mL})$. The fitting of the relative SANS profiles confirmed that the elongated shape of micellar aggregates for PEGylated valine octapeptide is maintained at all concentrations analysed above CAC (Figure 5B).
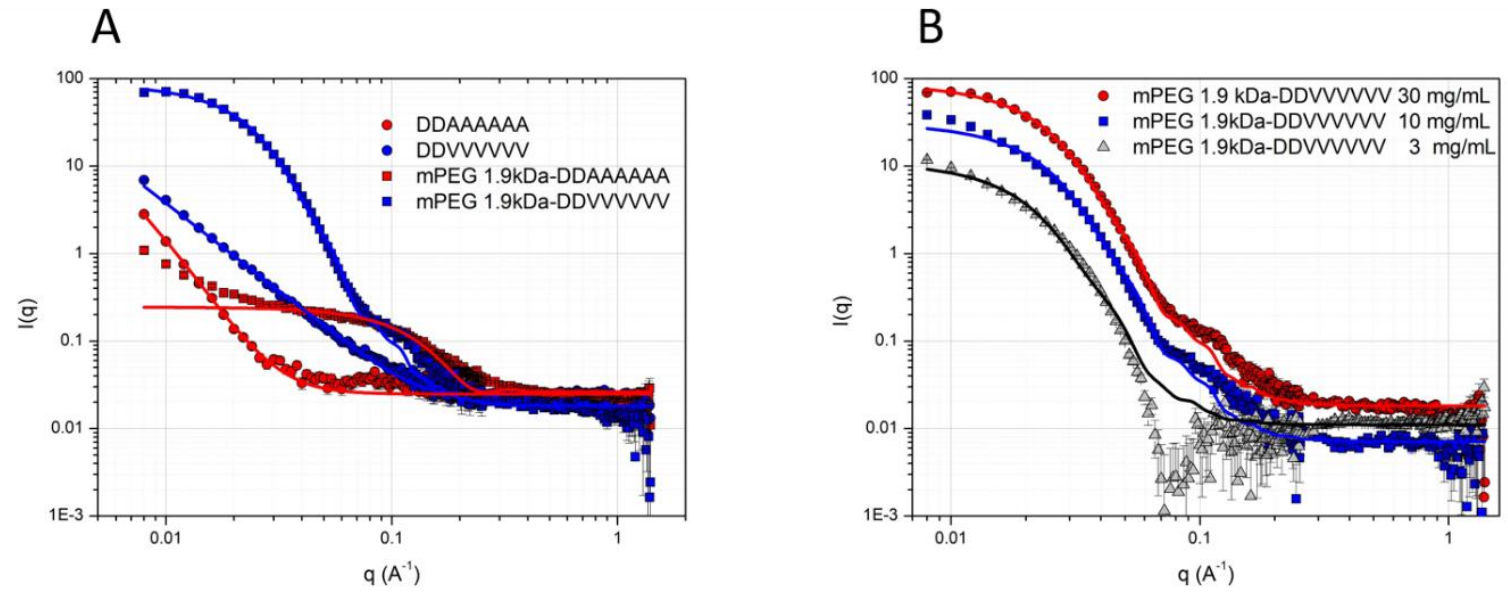

Figure 5 SANS profiles for non-PEGylated (DDAAAAAA and DDVVVVVV) octapeptides at a concentration of $5 \mathrm{mg} / \mathrm{mL}$ and for PEGylated $\left(\mathrm{mPEG}_{1.9 \mathrm{kDa}-\mathrm{DDAAAAAA}}\right.$ and $\mathrm{mPEG}_{1.9 \mathrm{kDa}^{-}}$ DDVVVVVV) octapeptides at a concentration of $30 \mathrm{mg} / \mathrm{mL}$ (A). SANS profiles for the $\mathrm{mPEG}_{1.9 \mathrm{kDa}^{-}}$ DDVVVVVV octapeptide at different concentrations above CAC $(30 \mathrm{mg} / \mathrm{mL}, 10 \mathrm{mg} / \mathrm{mL}$ and 3 $\mathrm{mg} / \mathrm{mL})(B)$. Lines represent the fitted signals.

Figure 6 shows the size distribution traces (intensity \%) relative to the analysed PEGylated or nonPEGylated amphiphilic DDVVVVVV peptide. A smaller and monomodal particle size distribution was observed for the PEGylated peptide, with a calculated hydrodynamic diameter of $26.63 \pm 0.41$ $\mathrm{nm}$. This size value is comparable to that of spherical/elongated micelles and comparable with the 
range of size $(10-50 \mathrm{~nm})$ reported for other PEGylated peptides ${ }^{18,19}$. On the other hand, bimodal and larger distributions were found for the non-PEGylated alanine based-peptide. The bimodal appearance of DLS traces related to non-PEGylated amphiphilic peptides (DDVVVVVV) reflects the possible non-spherical shape of these samples. DLS analysis in fact suggests the presence of at least two dimensions, one significantly below $100 \mathrm{~nm}$ (approximately 35-40 nm) and another from $200 \mathrm{~nm}$ to more than $1000 \mathrm{~nm}$. This result can be explained by considering DDVVVVVV could form fibrillary supramolecular structures, as reported in the literature for similar amphiphilic peptides ${ }^{20,21}$.

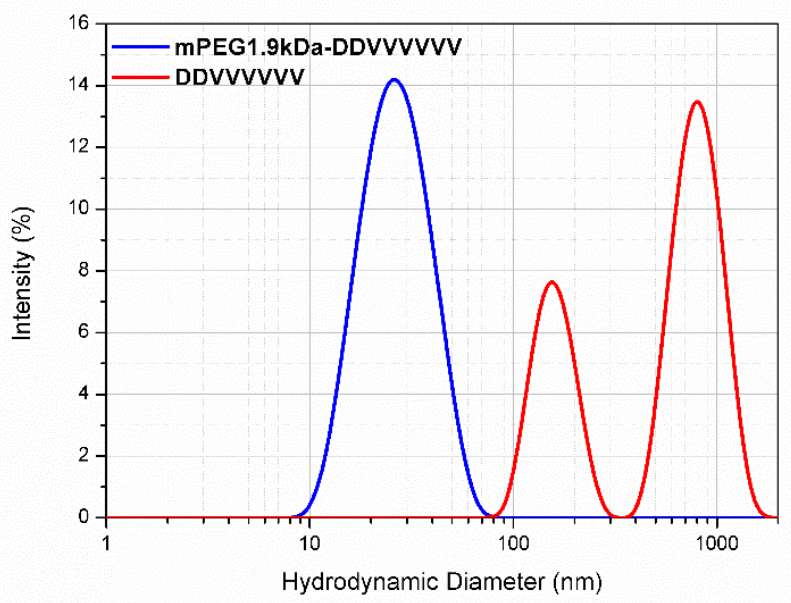

Figure 6 DLS traces of valine-based amphiphilic octapeptide at $25^{\circ} \mathrm{C}$ before and after PEGylation obtained at a concentration above CAC $\left(5 \mathrm{mg} / \mathrm{mL}\right.$ for DDVVVVVV and $30 \mathrm{mg} / \mathrm{mL}$ for $\mathrm{mPEG}_{1.9 \mathrm{ka}^{-}}$ DDVVVVVV).

Surfactant-like peptides represent a class of amphiphilic macromolecules, which have been widely characterized in terms of self-assembling behaviour. Amphiphilic peptides can form a number of supramolecular structures in water (e.g. nanofibers, nanorods, nanovesicles) due to different possible interactions between amino acid residues. Hydrogen bonds, van der Waals forces as well as electrostatic, hydrophobic and/or aromatic interactions ( $\pi-\pi$ stacking) can all be involved in the selfassembling process. The properties of these peptides can be tuned by changing the amino acid sequence or by a further derivatization at the amino or carboxyl end groups, leading to the design of novel functional biomaterials. In the field of drug delivery, peptides have been largely investigated as potential macromolecules for the design of novel nanocarriers, since they display some advantages over common synthetic surfactants. These include a better biocompatibility and the possibility of inducing specific biological functions or controlling drug release and enhancing cell uptake in response to stimuli, based on the amino acid sequence. 
The drawbacks of nanosystems formed by the self-assembly of surfactant-like peptides however include low stability in solution, poor aqueous solubility, potential heterogenicity of the nanoassemblies and the low drug loading. To overcome some limitations of these nanocarriers, hydrophilic peptides have been functionalized with fatty acids, to give rise to amphiphilic macromolecules, known as peptide amphiphiles, which, at the moment, represent the most investigated building blocks for the design of peptide-based nanocarriers.

Additional functionalization strategies of peptides are possible, which maintain the amphiphilic character of these materials and the self-assembly ability. One of these is PEGylation. Covering the surface of nanosystems with PEG is, indeed, a very common strategy to reduce immunogenicity, thereby increasing the systemic circulation time. The use of PEGylated lipids to prepare PEGylated liposomes (the so-called second generation or stealth liposomes) is an established practice. PEGylation has also been demonstrated as an effective approach to improve the stability and solubility of peptides in water. Despite these advantages, not enough attention has been focused on the effect of PEGylation on the self-assembling behaviour.

Self-assembling is known to be affected by the secondary structure (e.g. $\alpha$-helix, $\beta$-sheets) and the obtained nanostructures (such as nanospheres, nanofibers, nanorods and nanotubes) are dependent on the feature of the hydrophilic and hydrophobic portions of the material ${ }^{22}$. Different works have investigated the secondary structure of octapeptides ${ }^{23,24}$. Particularly for surfactant-like peptides formed by six hydrophobic and one or two anionic residues, a $\beta$-sheet conformation has been reported ${ }^{21}$, which is probably related to the repetition of the hydrophobic residues such as alanine or valine, owing to the stacking interaction via intermolecular hydrogen bonds ${ }^{3,25}$. In this work, a $\beta$-sheet conformation was observed for DDAAAAAA and DDVVVVVV peptides, which was not affected after conjugation with PEG. Actually, the effect of PEGylation on conformational stability of proteins or peptides is not well clarified ${ }^{26}$. In some studies, no effect was observed on the secondary structure of epta- or octapeptides after PEGylation ${ }^{27}$. No systematic studies can also be found in the literature in which CAC of PEGylated peptides are compared with that of non-PEGylated counterparts. Theoretically, PEGylation should increase the CAC value of peptides by increasing its hydrophilicity. For instance, CAC values in the range of $\mu \mathrm{M}$ up to 1-2 $\mathrm{mM}$ have been calculated for surfactant-like peptides ${ }^{28-30}$, and, generally, higher CAC values (in the range of $\mathrm{mM}$ ) have been found for PEGylated peptides ${ }^{31,11}$. SANS profiles confirmed the effect exerted by the coupling of $1.9 \mathrm{kDa}$ PEG to the hydrophilic portion on the self-assembling behaviour of the investigated surfactant-like octapeptides. We demonstrate that, PEGylation maintains the self-assembling properties only for the valine-based octapeptide. This is different to the effect seen with alanine-based peptides, suggesting that the coupling with $1.9 \mathrm{kDa}$ PEG confers a high level of hydrophilicity, which can be balanced only by the 
higher hydrophobicity of valine compared to alanine residues. Moreover, PEGylation seems to induce the formation of more homogeneous and elongated micelles, unlike non-PEGylated octapeptides for which fibrillar or ribbon-like structures have been already reported ${ }^{32-34}$.

\section{CONCLUSIONS}

Overall, the results suggest that the PEGylation of amphiphilic alanine- or valine-based octapeptides markedly affects their self-assembling behaviour in terms of critical aggregation concentration as well as in terms of shape and structure of the formed supramolecular aggregates. Particularly, PEGylation increases the CAC of these amphiphilic peptides and prevents the self-aggregation into fibrillary aggregates. As evidenced by SANS and DLS, PEGylation promotes the formation of elongated micellar structure for valine-based amphiphilic octapeptides, differently from the alaninebased amphiphilic octapeptide, which does not self-assemble at the concentrations tested (up to 30 $\mathrm{mg} / \mathrm{mL}$ ). Conversely, PEGylation does not influence the secondary conformation of amphiphilic octapeptides as evidenced by circular dichroism. Overall, these results suggest that PEGylation can modify the self-assembling behaviour of amphiphilic octapeptides, leading to the formation of different supramolecular aggregates. These findings can be helpful to enlarge the future applications of these nanomaterials in different fields.

\section{AUTHOR INFORMATION}

Corresponding author

*E-mail: 1uca.casettari@uniurb.it

\section{ORCID}

Diego Romano Perinelli: 0000-0002-7686-4150

Mario Campana: 0000-0002-2151-4110

Ishwar Singh: 0000-0001-7822-1063

Driton Vllasaliu: 0000-0002-4379-6171

Giovanni Filippo Palmieri: 0000-0002-5349-4978 


\section{ACKNOWLEDGMENT}

SANS experiments at the ISIS Neutron and Muon Source were supported by a beamtime allocation from the Science and Technology Facilities Council (UK).

The authors wish to thank Fabio De Belvis for the design and realization of the graphical abstract.

\section{REFERENCES}

(1) Akter, N.; Radiman, S.; Mohamed, F.; Reza, M. Self-Assembled Potential Bio Nanocarriers for Drug Delivery. Mini-Reviews Med. Chem. 2013, 13, 1327-1339.

(2) Zhao, X. Design of Self-Assembling Surfactant-like Peptides and Their Applications. Curr. Opin. Colloid Interface Sci. 2009, 14, 340-348.

(3) Paramonov, S. E.; Jun, H.-W.; Hartgerink, J. D. Self-Assembly of Peptide-Amphiphile Nanofibers: The Roles of Hydrogen Bonding and Amphiphilic Packing. J. Am. Chem. Soc. 2006, 128, 7291-7298.

(4) Vauthey, S.; Santoso, S.; Gong, H.; Watson, N.; Zhang, S. Molecular Self-Assembly of Surfactant-like Peptides to Form Nanotubes and Nanovesicles. Proc. Natl. Acad. Sci. U. S. A. 2002, 99, 5355-5360.

(5) Pashuck, E. T.; Cui, H.; Stupp, S. I. Tuning Supramolecular Rigidity of Peptide Fibers through Molecular Structure. J. Am. Chem. Soc. 2010, 132, 6041-6046.

(6) Hamley, I. W. PEG-Peptide Conjugates. Biomacromolecules 2014, 15, 1543-1559.

(7) Castelletto, V.; Hamley, I. W. Self Assembly of a Model Amphiphilic Phenylalanine Peptide/Polyethylene Glycol Block Copolymer in Aqueous Solution. Biophys. Chem. 2009, $141,169-174$.

(8) Tzokova, N.; Fernyhough, C. M.; Topham, P. D.; Sandon, N.; Adams, D. J.; Butler, M. F.; Armes, S. P.; Ryan, A. J. Soft Hydrogels from Nanotubes of Poly(Ethylene Oxide)?Tetraphenylalanine Conjugates Prepared by Click Chemistry. Langmuir 2009, 25, 2479-2485.

(9) Tzokova, N.; Fernyhough, C. M.; Butler, M. F.; Armes, S. P.; Ryan, A. J.; Topham, P. D.; Adams, D. J. The Effect of PEO Length on the Self-Assembly of Poly(Ethylene Oxide)?Tetrapeptide Conjugates Prepared by "Click" Chemistry. Langmuir 2009, 25 , 11082-11089.

(10) Diaferia, C.; Mercurio, F. A.; Giannini, C.; Sibillano, T.; Morelli, G.; Leone, M.; Accardo, A. Self-Assembly of PEGylated Tetra-Phenylalanine Derivatives: Structural Insights from 
Solution and Solid State Studies. Sci. Rep. 2016, 6, 26638.

(11) Dong, H.; Dube, N.; Shu, J. Y.; Seo, J. W.; Mahakian, L. M.; Ferrara, K. W.; Xu, T. LongCirculating $15 \mathrm{Nm}$ Micelles Based on Amphiphilic 3-Helix Peptide-PEG Conjugates. ACS Nano 2012, 6, 5320-5329.

(12) Hollamby, M. J. Practical Applications of Small-Angle Neutron Scattering. Phys. Chem. Chem. Phys. 2013, 15, 10566.

(13) Heenan, R. K.; Penfold, J.; King, S. M. SANS at Pulsed Neutron Sources: Present and Future Prospects. J. Appl. Cryst 1997, 30, 1140-1147.

(14) Arnold, O.; Bilheux, J. C.; Borreguero, J. M.; Buts, A.; Campbell, S. I.; Chapon, L.; Doucet, M.; Draper, N.; Ferraz Leal, R.; Gigg, M. A.; et al. Mantid-Data Analysis and Visualization Package for Neutron Scattering and $\mu$ SR Experiments. Nucl. Instruments Methods Phys. Res. Sect. A Accel. Spectrometers, Detect. Assoc. Equip. 2014, 764, 156-166.

(15) Blondelle, S. E.; Behrouz Forood; Richard A. Houghten, A.; Pérez-Payá, E. PolyalanineBased Peptides as Models for Self-Associated $\beta$-Pleated-Sheet Complexes. 1997, 36, 83938400 .

(16) Cheng, P.-N.; Pham, J. D.; Nowick, J. S. The Supramolecular Chemistry of $\beta$-Sheets. J. Am. Chem. Soc. 2013, 135, 5477.

(17) Naito, A.; Okushita, K.; Nishimura, K.; Boutis, G. S.; Aoki, A.; Asakura, T. Quantitative Analysis of Solid-State Homonuclear Correlation Spectra of Antiparallel $\beta$-Sheet Alanine Tetramers. J. Phys. Chem. B 2018.

(18) Dong, H.; Dube, N.; Shu, J. Y.; Seo, J. W.; Mahakian, L. M.; Ferrara, K. W.; Xu, T. LongCirculating $15 \mathrm{Nm}$ Micelles Based on Amphiphilic 3-Helix Peptide-PEG Conjugates. ACS Nano 2012, 6, 5320-5329.

(19) Diaferia, C.; Mercurio, F. A.; Giannini, C.; Sibillano, T.; Morelli, G.; Leone, M.; Accardo, A. Self-Assembly of PEGylated Tetra-Phenylalanine Derivatives: Structural Insights from Solution and Solid State Studies. Sci. Rep. 2016, 6, 26638.

(20) Qiu, F.; Chen, Y.; Tang, C.; Zhang, J.; Gong, M.; Su, B. Self-Assembling Surfactant-like Peptide A6K as Potential Delivery System for Hydrophobic Drugs. Int. J. Nanomedicine 2015, $10,847$.

(21) Adams, D. J.; Holtzmann, K.; Schneider, C.; Butler, M. F. Self-Assembly of Surfactant-like Peptides. Langmuir 2007, 23, 12729-12736.

(22) Song, Z.; Chen, X.; You, X.; Huang, K.; Dhinakar, A.; Gu, Z.; Wu, J. Self-Assembly of Peptide Amphiphiles for Drug Delivery: The Role of Peptide Primary and Secondary Structures. Biomater. Sci. 2017, 5, 2369-2380.

(23) Capes, J. S.; Kiley, P. J.; Windle, A. H. Investigating the Effect of PH on the Aggregation of Two Surfactant-Like Octapeptides. Langmuir 2010, 26, 5637-5644.

(24) Novelli, F.; De Santis, S.; Diociaiuti, M.; Giordano, C.; Morosetti, S.; Punzi, P.; Sciubba, F.; Viali, V.; Masci, G.; Scipioni, A. Curcumin Loaded Nanocarriers Obtained by SelfAssembly of a Linear d,l-Octapeptide-Poly(Ethylene Glycol) Conjugate. Eur. Polym. J. 2018, 98, 28-38.

(25) Castelletto, V.; Gouveia, R. J.; Connon, C. J.; Hamley, I. W.; Seitsonen, J.; Ruokolainen, J.; Longo, E.; Siligardi, G. Influence of Elastase on Alanine-Rich Peptide Hydrogels. Biomater. Sci. 2014, 2, 867-874. 
(26) Lawrence, P. B.; Price, J. L. How PEGylation Influences Protein Conformational Stability. Curr. Opin. Chem. Biol. 2016, 34, 88-94.

(27) Castelletto, V.; Newby, G. E.; Hermida Merino, D.; Hamley, I. W.; Liu, D.; Noirez, L. SelfAssembly of an Amyloid Peptide Fragment-PEG Conjugate: Lyotropic Phase Formation and Influence of PEG Crystallization. Polym. Chem. 2010, 1, 453.

(28) Yang, S. J.; Zhang, S. Self-Assembling Behavior of Designer Lipid-like Peptides. Supramol. Chem. 2006, 18, 389-396.

(29) Nagai, A.; Nagai, Y.; Qu, H.; Zhang, S. Dynamic Behaviors of Lipid-like Self-Assembling Peptide A6D and A6K Nanotubes. J. Nanosci. Nanotechnol. 2007, 7, 2246-2252.

(30) Wang, J.; Han, S.; Meng, G.; Xu, H.; Xia, D.; Zhao, X.; Schweins, R.; Lu, J. R. Dynamic Self-Assembly of Surfactant-like Peptides A6K and A9K. Soft Matter 2009, 5, 3870.

(31) Lee, E. S.; Shin, H. J.; Na, K.; Bae, Y. H. Poly(1-Histidine)-PEG Block Copolymer Micelles and PH-Induced Destabilization. J. Control. Release 2003, 90, 363-374.

(32) Valéry, C.; Paternostre, M.; Robert, B.; Gulik-Krzywicki, T.; Narayanan, T.; Dedieu, J.-C.; Keller, G.; Torres, M.-L.; Cherif-Cheikh, R.; Calvo, P.; et al. Biomimetic Organization: Octapeptide Self-Assembly into Nanotubes of Viral Capsid-like Dimension. Proc. Natl. Acad. Sci. U. S. A. 2003, 100, 10258-10262.

(33) Mandal, D.; Nasrolahi Shirazi, A.; Parang, K. Self-Assembly of Peptides to Nanostructures. Org. Biomol. Chem. 2014, 12, 3544-3561.

(34) Qiu, F.; Chen, Y.; Tang, C.; Zhao, X. Amphiphilic Peptides as Novel Nanomaterials: Design, Self-Assembly and Application. Int. J. Nanomedicine 2018, 13, 5003-5022. 